\title{
Synthese des Gentisins
}

ron

\author{
St. v. Kostanecki und J. Tambor.
}

Aus dem chemischen Laboratorium der Universität Bern.

(Vorgelegt in der Sitzung am 16. November 1893.)

Vor zwei Jahren hat der Eine von uns ${ }^{1}$ mitgetheilt, dass der Farbstoff der Enzianwurzel, das Gentisin $\mathrm{C}_{14} \mathrm{H}_{10} \mathrm{O}_{5}$, beim $\mathrm{Kc}$ 'hen mit Jodwasserstoffsäure $(1 \cdot 7)$ entmethylirt wird und eine neue Verbindung das Gentisein $\mathrm{C}_{13} \mathrm{H}_{8} \mathrm{O}_{5}$ liefert. Das Gentisin wäre also der Monomethyläther des Gentiseins, welche Auffassung darin eine Bestätigung findet, dass im Gentisein durch die Acetylirungsmethode drei Hydroxyle nachweisbar sind, während aus dem Gentisin, wie Hlasiwet $z$ und Habermann ${ }^{2}$ gefunden haben, nur ein Diacetylproduct erhalten wird. Kurz nacher haben Schmidt und der Eine von uns" den Beweis hiefür geliefert, indem sowohl das Gentisin als auch das Gentisein beim Methyliren dasselbe Endproduct, den Gentisinmonomethyläther ergaben.

Die Identität des Gentisinmonomethyläthers und des Gentiseindimethyläthers wurde durch directe Vergleichung und durch Darstellung ihrer Acetylverbindungen festgestellt. Wir haben noch die Benzoylverbindungen der beiden Äther erhalten und bei diesen Derivaten ebenfalls vollständige Übereinstimmung gefunden.

1 Monatshefte für Chemie, 12, 205

2 Liebig's Annalen, 175, 74.

3 Monatshefte für Chemie, 12, 318. 


\section{Benzoyl-Gentisinmonomethyläther (Benzoyl-Gentisein- dimethyläther) $\mathrm{C}_{13} \mathrm{H}_{5} \mathrm{O}_{2}\left(\mathrm{OCH}_{3}\right)_{2}\left(\mathrm{OCOC}_{6} \mathrm{H}_{5}\right)$.}

Versetzt man eine alkoholische Lösung des Centisinmethyläthers mit verdünnter Natronlauge, so erhält man ein schwer lösliches, gelbes Natronsalz. Dasselbe wurde abfiltrirt, ausgewaschen, in verdünnter Natronlauge suspendirt und mit Benzoylchlorid versetzt. Beim starken Schütteln verschwand die gelbe Farbe des Salzes fast vollständig und es entstand ein weisser, in Natronlauge unlöslicher Niederschlag, der aus siedendem Eisessig umkrystallisirt wurde. Wir erhielten so aus beiden Äthern weisse, glänzende, büschelförmig vereinigte Nadeln, die denselben Schmelzpunkt $\left(197^{\circ}\right)$ besassen. Die Benzoylverbindung des Gentiseindimethy äthers wurde analysirt: $0.2051 \mathrm{~g}$ Substanz gaben $0.5248 g \mathrm{CO}_{2}$ und $0.0785 \mathrm{~g} \mathrm{H}_{2} \mathrm{O}$.

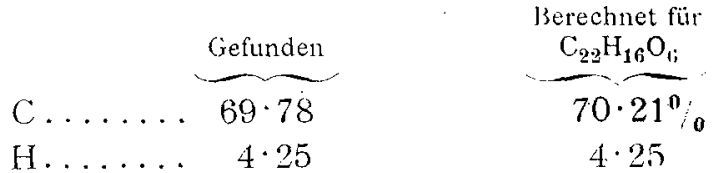

Nach der Feststellung der Beziehung der beiden in Rede stehenden Farbstoffe zu einander war die Frage nach der Constitution des Gentisins mit der vollständigen Erforschung des Gentiseins auf's Engste verknüpft. Das letztere erschien uns deswegen einer näheren Untersuchung zugänglich, als es, wie bereits früher hervorgehoben, die grössten Analogien mit den Oxyxanthonen zeigte und seiner Zusammensetzung nach ein Trioxyxanthon sein konnte. Schmidt und der Eine von uns haben auch erwähnt, dass man sich auf Grund der Thatsache, dass es bei der Kalischmelze in Hydrochinoncarbonsäure und Phloroglucin zerfällt, eine Vorstellung über die Lagerung der Hydroxylgruppen machen könnte, so dass dem Gentisein die Constitution

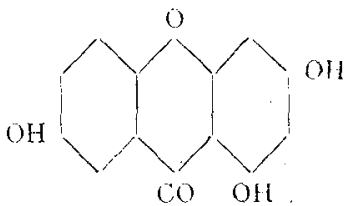

zukommen würde. 
Wenn auch der Befund, dass das Gentisein Thonerdebeize anfärbt, gegen die obige Formel, die keine Hydroxylgruppen in Orthostellung enthält, zu sprechen schien, so war es doch geboten, die Paarung der Hydrochinoncarbonsäure mit Phloroglucin $z u$ einem Trioxyxanthon zu untersuchen und die entstandene Verbindung mit dem Gentisein zu vergleichen. Da die Hydrochinoncarbonsäure die Xanthoncondensation bei der Copulirung mit Resorcin (Euxanthonsynthese) ohne Schwierigkeiten eingeht und das Phloroglucin bei der Paarung mit Salicylsäure und ihren Homologen von allen Phenolen die besten Ausbeuten liefert, so glaubten wir, aus den erwähnten Componenten leicht das gewünschte Trioxyxanthon erhalten zu können.

Der Versuch zeigte aber, dass wir uns in unseren Erwartungen arg getäuscht hatten, indem die ersten Destillationen nur Spuren eines gelbgefärbten Sublimats ergaben, während fast die gesammte Reactionsmasse als kohliger Rückstand zurückblieb. Da wir jedoch mit der geringen Menge der erhaltenen gelben Nadeln bei der Behandlung mit Natriumamalgam dieselbe Färbung wie beim Gentisein erhielten, so musste die Darstellung dieses Trioxyxanthons in einer zur Untersuchung hinreichenden Menge um so nothwendiger erscheinen.

Alle Versuche, das Essigsäureanhydrid durch ein anderes wasserentziehendes Mittel zu ersetzen, blieben erfoiglos.

Es blieb uns nichts übrig, als der Versuch, die zuerst gewählte Methode so auszuarbeiten, dass wesentlich grössere Ausbeuten zu erwarten wären. Von der Vermuthung ausgehend, dass möglicherweise gerade die Tendenz des Phloroglucins mit Salicylsäuren Xanthoncondensationen einzugehen, bei der Paarung mit der Gentisinsäure die Entstehung von Oxydixanthonen verursacht, die bei der Destillation, da sie schwer sublimiren, verkohlen und die Ausbeute beeinträchtigen, wendeten wir nur so viel Essigsäureanbydrid an, als gerade zur J.ösung der beiden Substanzen genügte. Es zeigte sich aber bald, dass diese Vorsicht allein nicht genügt, man muss vielmehr ausserdem noch den Gang der Destillation mit besonderer Sorgfalt leiten. Die besten Bedingungen sind, unseren Beobachtungen nach, die folgenden: 
Gentisinsäure und Phloroglucin werden in äquimolecularer Menge innig gemischt und mit der zur Lösung nöthigen Menge Essigsäureanhydrid versetzt. Es ist zweckmässig, nur wenige Gramme des Gemisches auf einmal in kleinen Retorten zu verarbeiten. Man destillirt das Essigsüureanhydrid so rasch ais möglich ab, und sobald die dunkle Masse dickflüssig geworden ist, erhitzt man die Retorte mehrere Stunden lang mit sehr kleiner Flamme. Allmälig setzen sich im Retortenhalse kleine, zu Büscheln vereinigte, gelbe Nädelchen an, die mit Alkohol aufgrenommen werden.

Nach dem Einengen der Lösung und Wasserzusatz bis zur beginnenden Trübung erhielten wir einen Krystallbrei, der indessen unter dem Mikroskop kein einheitliches Aussehen yeigte. Wahrscheinlich waren den Krystallen des Trioxyxanthons noch Spuren von Acetyl-Hydrochinon und AcetylPhloroglucin beigemischt. Um eine Trennung von diesen Acetylderivaten zu bewirken, wurde das Product in Alkali aufgelöst, filtrirt und mit Salzsäure gefällt. Diese Reinigungsmethode hat aber den Nachtheil, dass das Acetyl-Hydrochinon bei Gegenwart von Alkali durch den Sauerstoff der Luft oxydirt wird und schwer zu entfernende Verunreinigungen liefert. Der erhaltene Niederschlag war stets schwach bräunlich gefärbt, trotzdem wir, um die Oxydation des Hydrochinons zu verhüten, die alkalische Lösung rasch filtrirten und sie sofort in ver. dünnte Salzsäure einfliessen liessen. Einige Vorversuche belehrten uns bald, dass bei der geringen Menge der Substanz eine Reinigung durch Umkrystallisiren nicht leicht zu erzielen war. Wir zogen daher die sichere, wenn auch verlustreiche Reinigungsmethode - die Sublimation - vor. Dieselbe wurde mit kleinen Quantitäten der scharf getrockneten Substanz zwischen zwei Uhrgläsern ausgeführt. Das gesammelte Sublimat ergab alsdann nach zweimaligem Umkrystallisiren aus verdünntem Alkohol kleine, $z u$ Gruppen vereinigte Nädelchen, die sehr schwach gelb gefärbt waren und denselben Schmelzpunkt $\left(315^{\circ}\right)$ wie das aus Gentisin dargestellte Gentisein besassen.

Die Natriumamalgamreaction und dic Thatsache, dass dieses 'Trioxyxanthon Thonerdebeize gelb anfärbte, liessen keinen Zweifel.übrig, dass hier in der That das Gentisein vorlag. 
Um jedoch die Identität streng.zu beweisen, war die Bestimmung des Krystallwassers erwünscht, da dieses gerade für das Gentisein ausserordentlich charakteristisch ist.

Trotzdem die Ausbeute an dem Destillate bedeutend schlechter als bei allen bisher im hiesigen Laboratorium dargestellten Oxyxanthonen ist, und die umständliche Reinigungsmethode noch mit starken Verlusten verbunden ist, gelang es uns schliesslich, $0.4 g$ des reinen Gentiseins zu erhalten. Wir mussten aber eine grosse Anzahl von Retorten aufstellen, da die Ausbeute an dem reinen Producte unter $0.2 \%$ der angewandten Gentisinsäure ist.

$0 \cdot 4008 g$ Substanz verloren beim Erhitzen auf $110^{\circ} 0.0528 g \mathrm{H}_{2} \mathrm{O}$.

$$
\mathrm{H}_{2} \mathrm{O} \ldots \ldots \underbrace{\text { Gefunden }}_{12 \cdot 67} \frac{\begin{array}{c}
\text { Berechnet für } \\
\mathrm{C}_{13} \mathrm{H}_{8} \mathrm{O}_{5}+2 \mathrm{H}_{2} \mathrm{O}
\end{array}}{12 \cdot 86 \%}
$$

Die so getrocknete Substanz gab bei der Analyse folgende Zahlen:

$0 \cdot 1889 g$ Substanz gaben $0 \cdot 4456 g \mathrm{CO}_{2}$ und $0 \cdot 0626 g \mathrm{H}_{2} \mathrm{O}$.

$$
\begin{aligned}
& \text { Berechnet für } \\
& \text { Gefunden } \\
& \text { C....6 64.33 } \\
& \text { H..... } 3 \cdot 17 \\
& \mathrm{C}_{13} \mathrm{H}_{8} \mathrm{O}_{5} \\
& 63 \cdot 93 \% \\
& 3 \cdot 28
\end{aligned}
$$

Die bei der Krystallwasserbestimmung des natürlichen Gentiseins früher gefundenen Zahlen sind $12 \cdot 87 \%$, somit findet auch in diesem Punkte vollständige Übereinstimmung statt. Zum Überfluss haben wir noch die Acetylverbindung dargestellt und den richtigen Schmelzpunkt (226 ) gefunden.

Durch die Synthese des Gentiseins ist seine Formel unzweideutig festgestellt, weswegen man nun diesen Namen fallen lassen kann; es ist das 1-, 3-, 7-Trioxyxanthon:

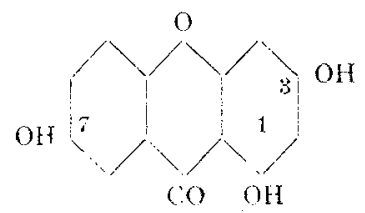


Da das Gentisein, wie nun bewiesen ist, keine Hydroxylgruppen in der Orthostellung enthält, so sollte man erwarten, tass es sich indifferent gegen Beizen verhalten würde. Es färbt indessen, wie bereits früher mitgetheilt, Thonerdebeize gelb an. Die Färbungen sind allerdings nicht intensiv, doch unverkennbar.

Diese Thatsache ist um so auffallender, als das 1-, 2-, 3-Monooxyxanthon, das Euxanthon, das 1-, 3-Isoeuxanthon und auch das Gentisin, Verbindungen, die die Hydroxyle in derselben Stellung nur in geringerer Anzahl enthalten, Beizen absolut nicht anzufärben vermögen.

Es ist vor der Hand nicht mit Bestimmtheit zu ersehen, wie man diese Thatsache mit der Theorie der beizenziehenden Farbstoffe in Einklang bringen kann. Dass die von dem Einen von uns hervorgehobenen Regelmässigkeiten thatsächlich existiren, beweisen nicht nur die in den erwähnten Mittheilungen angeführten Beispiele, sondern mehrere Berbachtungen, die nach der Aufstellung der Theorie über die beizenziehenden Farbstoffe sowohl in der wissenschaftlichen Literatur, als auch durch Mittheilungen aus der Praxis bekannt geworden sind. Indessen müssen wir darauf aufmerksam machen, dass unter den auf ihr Färbevermögen untersuchten Substanzen die nichtfärbenden Abkömmlinge in relativ geringerer Anzahl geprült worden sind. Das von der Regel abweichende Verhalten des Gentiseins lässt jedenfalls die Untersuchung polyhydroxylirter Farbstoffe, die keine Hydroxylgruppen in Orthostellung zu einander enthalten, wünschenswerth erscheinen.

Als nach den obigen Versuchen die Muttersubstan\% des Gentisins synthetisch dargestellt war, handelte es sich nun darum, diesen Pflanzenfarbstoff selbst, wie er in der Enzianwurzel vorkommt, künstlich zu erhalten. Der am nächsten liegende Weg war, durch partielle Methylirung in das Gentisein nur eine Methylgruppe einzuführen. Der Theorie nach können allerdings drei Monomethyläther entstehen:

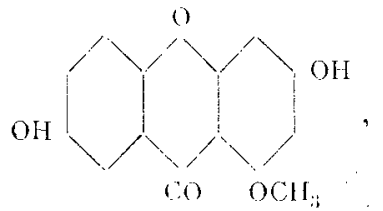

(C) OCH:;

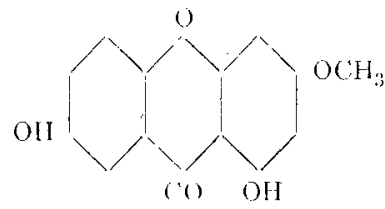


und

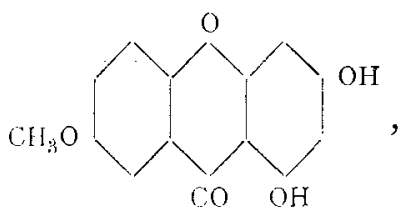

indessen war die Bildung nur zweier Ather zu erwarten, indem Dreher und der Eine von uns' gezeigt haben, dass das in der Orthostellung zum Carbonyl befindliche Hydroxyl bei den Oxyxanthonen nicht methylirt wird.

Der Versuch verlief recht günstig, da wir in dem Reactionsproducte nur e in en Monomethyläther auffinden konnten und derselbe sich mit dem natürlichen Gentisin identisch erwies.

Da die Beschaffung des synthetischen Gentiseins in der erforderlichen Menge mit grossen Schwierigkeiten verknüpft gewesen wäre, so waren wir gezwungen, das aus dem Gentisin durch Entmethyliren dargestelle Gentisein $z u$ diesem Versuche anzuwenden.

Dass aber dieses Gentisein absolut frei von Gentisin war, beweist die Krystallwasserbestimmung

$1 \cdot 319 g$ Substanz verloren beim Erhitzen aut $100^{\circ} 0 \cdot 1688 g \mathrm{H}_{2} \mathrm{O}$.

$$
\mathrm{H}_{2} \mathrm{O} \ldots \ldots+\frac{\text { Gefunden }}{12 \cdot 72 \%} \quad \frac{\begin{array}{c}
\text { Berechnct für } \\
\mathrm{C}_{13} \mathrm{H}_{8} \mathrm{O}_{5}+2 \mathrm{H}_{2} \mathrm{O}
\end{array}}{12 \cdot 86^{\circ} \%}
$$

1 Molekül des Gentiseins wurde nun mit 1 Molekül Kalihydrat und etwas mehr ais 1 Molekül Methyljodid in methylalkoholischer Lösung mehrere Stunden auf $100^{\circ}$ erhitzt, der Röhreninhalt bis zur Verjagung des Methylalkohols auf dem Wasserbade eingedampft und der Rückstand mit verdünnter Natronlauge aufgenommen. Hiebei ging der grösste Theil in Lösung, das Ungelöste bestand aus dem Natronsalze des Gentiseindimethyläthers. Die abfiltrirte Flüssigkeit gab mit Salzsäure einen schwach gelb gefärbten, flockigen Niederschlag, der behufs Reinigung von unangegriffenen Gentisein mehrmals

1 Ber. d. deutschen chem. Gesellschaft, $26,71$. 
aus Alkohol umkrystallisirt wurde. Die erhaltenen gelben Nädelchen besassen denselben Schmelzpunkt, wie das natürliche Gentisin $\left(267^{\circ}\right)$ und gaben mit Natriumamalgam dieselbe Farbenreaction. Nach der üblichen Methode acetylirt, wurden aus beiden Präparaten weisse Nädelchen erhalten, die den von Hlasiwetz und Habermann angegebenen Schmelzpunkt $\left(1965^{\circ}\right)$ besassen. Die Reinheit des synthetischen Acetyl. Gentisins haben wir noch durch eine Analyse controlirt.

$0.1954 g$ Substanz gaben $0.4533 g \mathrm{CO}_{2}$ und $0.0798 g \mathrm{H}_{2} \mathrm{O}$.

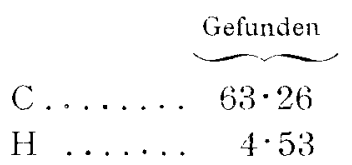

Berechnet für

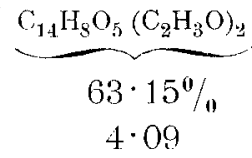

Schliesslich haben wir nach der Methode von Schotten ${ }^{1}$ die Benzoylverbindung des synthetischen Gentisins dargestellt. Das Reactionsproduct krystallisirte aus Alkohol in dicken, blendend weissen Nadeln, die bei $192^{\circ}$ schmolzen.

Die Benzoylverbindung des natürlichen Gentisins zeigte völlig dieselben Eigenschaften und ergah bei der Analyse:

$0.1974 g$ Substanz gaben $0.5192 \mathrm{~g} \mathrm{CO}$, und $0.0716 g \mathrm{H}_{2} \mathrm{O}$.

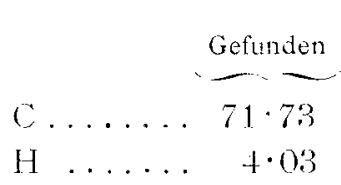

Berechnet für

$$
\begin{gathered}
\mathrm{C}_{11} \frac{\mathrm{H}_{8} \mathrm{O}_{5}\left(\mathrm{COC}_{6} \mathrm{H}_{5}\right)_{2}}{72 \cdot 10^{n} / 6} \\
3 \cdot 86
\end{gathered}
$$

Hiernach ist an der Identität des Gentiseinmonomethylathers mit dem natürlichen Gentisin wohl nicht zu zweifeln. Die Constitution des Gentisins bleibt aber trotz der Synthese noch nicht ganz aufgeklärt, denn zwischen den beiden möglichen Formeln (Formel II und III) lässt sich zur Zeit mit Sicherheit nicht entscheiden.

1 Ber. d. deutschen chem. Gesellschaft, XVII, 225, 45. 